\title{
FlexWork - \\ Lastregelung bei einem Motor mit elektrohydraulischem, vollvariablem Ventiltrieb
}

Norbert Zsiga, Empa, Swiss Federal Laboratories

for Materials Science and Technology

Andyn Omanovic, Empa

Patrik Soltic, Empa

Wolfgang Schneider, Wolfgang Schneider Ingenieurbüro

This document is the accepted manuscript version of the following article:

Zsiga, N., Omanovic, A., Soltic, P., \& Schneider, W. (2019).

FlexWork - Lastregelung bei einem Motor mit elektrohydraulischem, vol1variablem Ventiltrieb. In J. Lieb1 (Ed.), Proceedings. Ladungswechse 1 und Emissionierung 2018 Im Spannungsfeld von Luftqualität, Klimaschutz und Elektrifizierung. 11. MTZ-Fachtagung 


\section{Einleitung}

Niedrige $\mathrm{CO}_{2}$-Emissionen zu erreichen, ist ein wichtiges Ziel für alle Automobilhersteller, um massive Strafzahlungen zu verhindern. Ab 2020 gilt für neu zugelassene PKW ein Flottengrenzwert von 95g/km (für ein Referenzfahrzeug von $1372 \mathrm{~kg}$ ), für den Zeitraum bis 2030 wird aktuell eine weitere, herstellerspezifische, relative Grenzwertabsenkung diskutiert. Der Umstieg vom alten NEFZ- zum neuen WLTPPrüfverfahren erschwert das Erreichen der $\mathrm{CO}_{2}$-Grenzwerte zusätzlich. Daher ist der einzige Weg zur Minimierung der $\mathrm{CO}_{2}$-Emissionen bei Fahrzeugen mit Verbrennungsmotor eine Dekarbonisierung des Kraftstoffs und das konsequente Ausnutzen von Wirkungsgradpotenzialen.

Bei Ottomotoren wird unter anderem versucht das Wirkungsgradpotenzial durch Downsizing und Hybridisierung auszuschöpfen. In beiden Fällen sorgt die Verlagerung häufig durchfahrener Betriebspunkte in Kennfeldbereiche geringen spezifischen Verbrauchs für eine Reduktion des $\mathrm{CO}_{2}$-Ausstoßes. Beim Hybrid kann zusätzlich noch Energie zurückgewonnen statt an den Bremsen dissipiert werden [1]. Die Bereiche hohen spezifischen Verbrauchs liegen in der niedrigen Teillast [2], wo über Variabilität im Ventiltrieb die Verluste (vor allem Gaswechselverluste [3]) stark reduziert werden können.

Vollvariable Ventilsteuerungen am Einlass und am Auslass sind der Wunschtraum aller Motorenentwickler, da durch die gewonnenen Freiheitsgrade neue Lösungsansätze für bekannte Verbrennungsmotorenprobleme ermöglicht werden. So kann zum Beispiel der Ottomotor entdrosselt werden, indem die Lastregelung über den Hub und die Öffnungsdauer der Einlassventile anstelle des Einlassdrucks erfolgt. Durch die Wahl der Ventilüberschneidungsdauer kann die interne Abgasrückführung eingestellt werden [4]. Beides führt zu einer Wirkungsgradsteigerung im Teillastbetrieb.

Des Weiteren sind Änderungen der Takt-Zahl oder Zylinderabschaltung leicht realisierbar. Um auch hier ein Beispiel zu nennen: Die Last eines jeden Zylinders kann erhöht werden, wenn von 4-Takt auf 6- oder gar 8-Takt Betrieb umgestellt wird. Eine andere Möglichkeit die spezifische Last zu erhöhen, bietet das temporäre Downsizing, welches über das Abschalten einzelner Zylinder oder Zylindergruppen erreicht werden kann [5].

Sofern die Steuerzeiten schnell angepasst werden können, ist eine sehr schnelle Änderung des Drehmoments ebenfalls möglich. Eine Optimierung der Volllastlinie ist durch Anpassung der Steuerzeiten und Ventilhübe über den gesamten Drehzahlbereich möglich. Zur Steigerung der Leistungsdichte ist eine Umschaltung auf 2-Takt Betrieb ebenfalls denkbar, sofern eine ausreichende Kopfumkehrspülung sowie ein entsprechendes Spülgefälle realisiert werden können. 
FlexWork - Lastregelung bei einem Motor mit elektrohydraulischem, vollvariablem ...

Neben diesen vielfältigen Maßnahmen zur Optimierung von Wirkungsgrad, Dynamik und Leistungsdichte ist es mittels vollvariabler Ventilsteuerung auch möglich, den Ausstoß von Schadstoffen zu reduzieren. So können beispielsweise beim Kaltstart die Auslassventile extrem früh geöffnet werden, so dass ein möglichst großer Teil der während der Verbrennung freigesetzten Wärme zum Aufwärmen des Katalysators genutzt werden kann. Bei vollvariablen Ventiltrieben bleibt die Ventilüberschneidungsphase durch diese Maßnahme unbeeinträchtigt.

Um die Vorteile eines vollvariablen Ventiltriebes voll ausnutzen zu können, muss der Ventiltrieb energetisch effizient, sprich in der Antriebsleistung mit einer konventionellen mechanischen Nockenwelle vergleichbar sein. Dies ist nicht trivial, denn ein Nockenwellentrieb ist in der Lage, einen großen Teil der beim Öffnungsvorgang in den Ventilfedern gespeicherten potenziellen Energie beim Schließvorgang wieder zu rekuperieren. Zudem sollten für den Betrieb keine komplexe Sensorik und Aktuatorik nötig sein. Neben einer großen Komplexität können hohe Kosten einen vollvariablen Ventiltrieb unattraktiv für die Serienproduktion machen. Viele in der Vergangenheit entwickelte, vollvariable Ventiltriebe haben den Weg in die Serienproduktion vermutlich aufgrund zu hohen Energiebedarfs oder zu hoher Systemkomplexität nicht gefunden.

Diese Veröffentlichung stellt einen elektrohydraulischen, vollvariablen Ventiltrieb („FlexWork“) vor, der an der Empa in enger Zusammenarbeit mit dem Wolfgang Schneider Ingenieurbüro entwickelt wurde. Das System wurde auf einem Versuchsmotor aufgebaut, bei dem sowohl einlass- als auch auslassseitig der Öffnungszeitpunkt und der Schließzeitpunkt der Ventile unabhängig voneinander und zylinderindividuell verstellt werden können. Der Ventilhub kann ebenfalls einalssseitg und auslassseitig unabhängig voneinander frei gewählt werden. Zunächst wird der Ventiltrieb, welcher ohne teure Materialien, Aktuatorik und Sensorik auskommt, vorgestellt. Einige Messergebnisse von einem Motorenprüfstand für den stationären und transienten Motorbetrieb folgen zur Illustration. Im Anschluss wird auf die Lastregelung unter Ausnutzung der Freiheitsgrade des vollvariablen Ventiltriebs, und die Art und Weise, wie Lastregelung und Energiebedarf des Ventiltriebs zusammenhängen, eingegangen. Schließlich wird gezeigt, dass der Energiebedarf des Ventiltriebs ähnlich dem eines konventionellen Ventiltriebs ist und in der Teillast sogar etwas geringer.

\section{FlexWork - Ein innovativer, vollvariabler Ventiltrieb}

Bei der Entwicklung eines vollvariablen Ventiltriebs müssen vor allem zwei Aspekte beachtet werden, Komplexität und Effizienz. Eine geringere Komplexität führt zu geringeren Herstellungskosten. Dazu gehören das Vermeiden von teurer Sensorik und das Minimieren der Anzahl Aktuatoren. Speziell bei der Entwicklung der hydraulischen Ventilkomponenten ist zudem wichtig, dass die Aktuatoren nicht bei hohen 
Strömungsgeschwindigkeiten schalten. Die bei Schaltvorgängen unvermeidlichen hydraulischen Drosselverluste ließen sich nur mittels extrem schnellen und damit teuren Aktuatoren klein halten, welche zudem einen hohen elektrischen Energiebedarf und Regelaufwand mit sich brächten. Des Weiteren muss das Gesamtsystem energetisch effizient sein, um mit den etablierten nockengetriebenen Ventiltrieben auf dem Markt konkurrieren zu können.

Die aufgeführten Ziele werden mit dem hier vorgestellten FlexWork Ventiltrieb erreicht, unter anderem dank einer seriellen Anordnung von Rückschlagventilen. Die Anzahl an Aktuatoren wurde gering gehalten, indem sowohl $\mathrm{Zu}$ - und Abfluss als auch die Rekuperation des Mediums über Rückschlagventile automatisch gesteuert werden. Somit werden keine externe Überwachung und Regelung der Fluidströmung oder des Ventilhubs benötigt. Außerdem führt eine hydraulische Bremse dazu, dass das Gaswechselventil auf den letzten Zehntelmillimetern automatisch abgebremst wird, um schließlich sanft aufzusetzen. Lediglich Beginn und Ende der Ventilöffnung werden über eine marktübliche Magnetspule gesteuert.

Eine weitere Besonderheit des hier präsentierten Ventiltriebs ist die eingesetzte Hydraulikflüssigkeit. Eine 50:50 Wasser-Ethylenglykol Mischung, also normales Motorkühlmittel, wird verwendet. Der Vorteil dieses Mediums liegt im höheren E-Modul von 28`000bar, verglichen mit Hydraulikölen mit einem E-Modul von 15‘000$20^{\circ} 000$ bar.

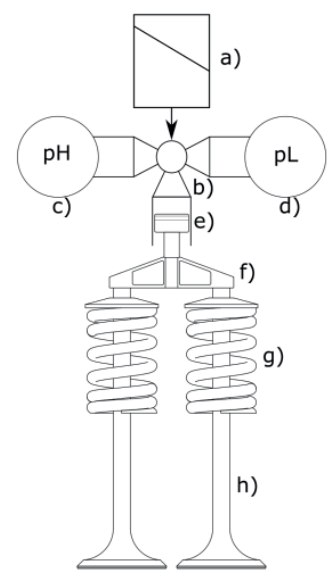

Abbildung 1: Schematische Darstellung des Ventiltriebs.
Ein höherer E-Modul führt zu einer Reduktion der kapazitiven hydraulischen Verluste. Über diesen Vorteil hinaus eröffnet ein komplett ölfreier Zylinderkopf auch die Möglichkeit, weniger stark additiviertes Motoröl zu verwenden, da die großen Flächenpressungen an den Nocken ausbleiben. Damit gelangen weniger toxische Schadstoffe in die Verbrennung und damit ins Abgas, was wiederum die Lebensdauer des Katalysators erhöhen oder die erforderliche Menge an Edelmetallen im Katalysator reduzieren kann. Grundsätzlich ließe sich der neue Ventiltrieb auch mit Motorenöl ohne hohe Anforderung an die Additivierung betreiben.

\section{Funktionsprinzip}

Der entwickelte Ventiltrieb ist in Abbildung 1 schematisch dargestellt. Das System beinhaltet einen Elektromagneten a), ein Schaltventil b), zwei Druckniveaus c) und d), und einen Arbeitskolben e). Der Ventiltrieb wur- 
de so ausgelegt, dass der Arbeitskolben auf eine Ventilbrücke f) drückt und dabei um die Komplexität gering zu halten - zwei Gaswechselventile h) öffnet. Der Schließvorgang erfolgt durch die beiden Federn der Gaswechselventile.

Der Bewegungsvorgang des Gaswechselventils lässt sich vereinfacht mit dem Modell eines Feder-Massen-Schwingers beschreiben. Um die Gaswechselventile zu öffnen, wird die Magnetspule a) bestromt, welche wiederum das hydraulische Schaltventil b) betätigt. Daraufhin strömt Hydraulikfluid vom Hochdruckniveau c) in den Arbeitsraum. Der sich dort aufbauende Druck wirkt auf den Arbeitskolben e), welcher sich daraufhin in Bewegung setzt.

In Abbildung 2 ist die hydraulische Kraft zusammen mit der Federkraft gegenüber dem Ventilhub für ein idealisiertes System aufgetragen. Bei einem unendlich langsamen Bewegungsvorgang würde sich bei der Kraft $F_{h y d r}$ ein statisches Gleichgewicht mit der Federkraft $F_{F}$

$$
F_{h y d r}\left(y_{G G}\right)=F_{F}\left(y_{G G}\right)=2 \cdot\left(q+y_{G G} \cdot k\right)
$$

einstellen, wobei q die Federvorspannkraft und $\mathrm{k}$ die Federkonstante einer Einzelfeder ist. Die Kraft $F_{\text {hydr }}$ hängt von dem gewählten Druck im Hochdruckniveau c) ab und muss über der Kraft der Federvorspannung q liegen.

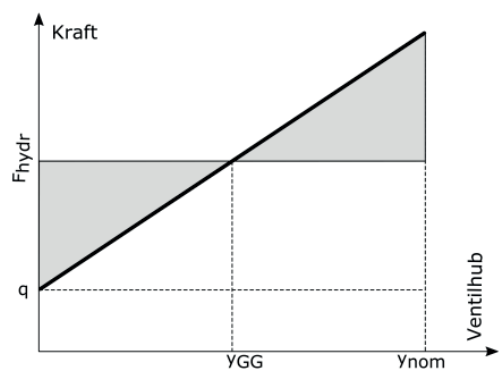

Abbildung 2: Schematische Darstellung der hydraulischen Rekuperation.

Der Überschuss an im Fluid im Hochdruckniveau c) gespeicherter Energie (dargestellt mit grauen Dreiecken) ermöglicht es, die Gaswechselventile stark zu beschleunigen. Dabei geht die im Druck gespeicherte Energie in kinetische Energie über. Die Ventile werden solange beschleunigt, bis die hydraulische Kraft und die Federkraft im Gleichgewichtspunkt $y_{G G}$ ausgeglichen sind. Bei $y_{G G}$ ist sämtliche im Druck gespeicherte Energie in kinetische Energie umgewandelt, was bedeutet, dass die Gaswechselventile die maximale Geschwindigkeit erreicht haben. Ab $y_{G G}$ werden die Gaswechselventile durch die grösser werdende Kraft der Gaswechselventilfedern abgebremst. Die kinetische Energie wird wieder in potenzielle Energie umgewandelt. Der nominelle Hub $y_{\text {nom }}$ ist erreicht, wenn sämtliche kinetische Energie als potenzielle Energie in den Gaswechselventilfedern gespeichert ist. Im verlustfreien Fall ist der nominelle Hub aufgrund der Energieerhaltung genau doppelt so groß wie der Hub im Gleichgewichtspunkt 
$y_{\text {nom }}=2 \cdot y_{G G}$. Mit dem Hochdruckniveau in c) wird demnach der zu erreichende Ventilhub $y_{\text {nom }}$ definiert.

Solange die Magnetspule bestromt ist, verhindern Rückschlagventile (in Abbildung 1 nicht dargestellt), dass das System zum Gleichgewichtspunkt $\mathrm{y}_{\mathrm{GG}}$ zurückschwingt. Dies hat zur Folge, dass sich aufgrund der Federkräfte im Arbeitsraum der doppelte Speisedruck einstellt, während die Gaswechselventile offen gehalten werden. Sobald die Magnetspule deaktiviert wird, wird das Rückschlagventil umgangen und die Ventilfedern drücken das Hydraulikfluid aus dem Arbeitsraum zurück in den Hochdruckspeicher (Rekuperation). In einem realen System treten kapazitive und dissipative Verluste auf, weshalb sich im Arbeitsraum nie der doppelte Speisedruck einstellt. Deswegen kann in Realität nie die gesamte Fluidmenge rekuperiert werden. Der Teil des Fluides, der nicht rekuperiert werden kann, wird zum Niederdruckniveau d) abgelassen. Kurz bevor die Gaswechselventile aufsetzen, wird der Ablassquerschnitt verringert (in Abbildung 1 nicht dargestellt), was eine Bremswirkung zur Folge hat und das Ventil sanft schließen lässt (Aufsetzbremse).

\section{Auslegung und Aufbau}

Da der Ventiltrieb als Feder-Massen-Schwinger beschrieben werden kann, hängt dessen Öffnungszeit $T_{\ddot{O} f f n u n g}$ von der Steifigkeit der Gaswechselventilfedern k und von der Masse aller bewegten Komponenten $M_{\text {System }}$ ab:

$$
T_{\text {Öffnung }}=\pi \cdot \sqrt{\frac{M_{\text {System }}}{k}} .
$$

Daraus ist ersichtlich, dass die Öffnungszeit unabhängig vom gewünschten Ventilhub und für alle Drehzahlen konstant ist. Die Steifigkeit der Gaswechselventilfedern wurde mit 45N/mm (je Feder) so gewählt, dass die Öffnungszeit für die ca. 100g schwere bewegte Masse beider Ventile inkl. Ventilbrücke $3 \mathrm{~ms}$ beträgt. Im Vergleich dazu: Konventionelle Nockenwellen dieser Baugröße arbeiten mit Federn, die eine Federsteifigkeit von ca. 16-19N/mm aufweisen.

Die Freiheitsgrade für die Systemauslegung betreffen einerseits die Öffnungs- und Schließzeit und andererseits das sanfte Aufsetzen der Ventile. Durch steifere Ventilfedern werden die Öffnungs-und Schließflanken steiler. Diese Anpassung führt dazu, dass höhere Hydraulikdrücke benötigt werden und der Energiebedarf der Hydraulik leicht ansteigt. Bei der Aufsetzbremse kann über die Positionierung eines Ablassschlitzes und den Durchmesser einer Düse eingestellt werden, ab welchem Hub die Bremswirkung einsetzt und wie groß die Schließgeschwindigkeit ist. Diese Parameter werden einmalig ausgelegt und bleiben dann bei allen Motordrehzahlen, Ventilhüben und Öffnungsdauern gleich. Das bedeutet, dass sich der Ventiltrieb bei hohen Dreh- 
FlexWork - Lastregelung bei einem Motor mit elektrohydraulischem, vollvariablem ...

zahlen ähnlich zu einem nockengetrieben Ventiltrieb verhält, dass sich jedoch für niedrige Motordrehzahlen sehr steile Gradienten über den Kurbelwinkel für Ventilöffnen und -schließen ergeben. Diese Eigenschaften führen dazu, dass sich im emissionsrelevanten niedrigen und mittleren Drehzahlbereich die gesuchte Vielzahl neuer Freiheitsgrade für die Wahl der Steuerzeiten ergibt.

Zusammenfassend resultiert also aus der Bestromungsdauer der Spule die Öffnungsdauer der Gaswechselventile, während der Ventilhub wird über das Hochdruckniveau bestimmt wird. Eine Feedback-Regelung, beispielsweise mittels Hubsensor, ist nicht zwingend erforderlich. Eine Ventilhubmessung kann aber selbstverständlich für Diagnosezwecke von Nutzen sein.

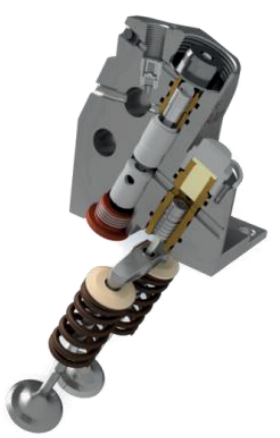

Abbildung 3: CAD

Rendering eines

Einlassmoduls mit

Gaswechselventilen.

Zur Fertigung der Komponenten wurden gewöhnliche korrosionsbeständige Stähle eingesetzt. Das Gehäuse und die Ventilbrücke wurden 5-Achsen CNC gefräst, die restlichen Teile sind Drehteile, wobei Steuerkanten aufgrund der geforderten Toleranzen drahterodiert wurden. Bei den Rückschlagventilen handelt es sich um marktübliche Zukaufteile. Laufbüchsen und Kolben wurden gehärtet, gehont respektive geschliffen und mit etwa $3 \mu \mathrm{m}$ Spiel gepaart. Solche präzisen Toleranzen sind notwendig, um Leckagen mit dem verwendeten, dünnflüssigen Hydraulikfluid $\mathrm{zu}$ minimieren. Die Laufbüchsen wurden zusätzlich kurzzeitnitriert um die Oberflächenhärte zu erhöhen und ein Anfressen des Kolbens zu vermeiden. Trotz der geringen Schmierfähigkeit der eingesetzten Wasser-Ethylenglykol Mischung wurden nach $50 \mathrm{Be}-$ triebsstunden noch keinerlei Gebrauchsspuren an Kolben oder Laufbüchsen festgestellt. Abbildung 3 zeigt das CAD Rendering eines Einlassmoduls (ohne Magnetspule).

\section{Mechanischer und elektrischer Energiebedarf}

Die benötigte mechanische Leistung eines Hydraulikmoduls im Betrieb in einem 4Takt Motor berechnet sich zu

$P_{h y d r}=\Delta p_{h y d r} \cdot \dot{V}_{h y d r}=\Delta p_{h y d r} \cdot \frac{\pi}{4} \cdot d_{A K}^{2} \cdot y \cdot \frac{1-\eta_{\text {Rek }}}{\eta_{\text {Pump }}} \cdot \frac{n_{\text {Mot }}}{2 \cdot 60}$.

Dabei ist $\Delta p_{\text {hydr }}$ die Druckdifferenz zwischen dem hohen und dem tiefen Druckniveau, $d_{A K}$ der Durchmesser des Arbeitskolbens, $y$ der maximale Hub während eines Ventilöffnungsvorgangs, $\eta_{\text {Rek }}$ die hydraulische Rekuperationsrate, $\eta_{\text {Pump }}$ der Wirkungsgrad der Hydraulikpumpe und $n_{M o t}$ die Motordrehzahl. Um den Reibmitteldruck auszudrücken, ändert sich obige Gleichung zu, 
$p_{m r}=\frac{P_{h y d r}}{\frac{n_{M o t}}{2 \cdot 60} \cdot V_{h}}=\Delta p_{h y d r} \cdot \frac{\pi}{4} \cdot d_{A K}^{2} \cdot y \cdot \frac{1-\eta_{R e k}}{\eta_{\text {Pump }}} \cdot \frac{1}{V_{h}}$.

Der Term $V_{h}$ ist das Hubvolumen des Motors, die Drehzahlabhängigkeit entfällt. Unter der Annahme, dass die Rekuperationsrate und die Pumpeneffizienz konstant sind und unter Berücksichtigung der Tatsache, dass der Ventilhub eine Funktion des Hochdruckniveaus ist, wird klar, dass der Reibmitteldruck lediglich vom Ventilhub abhängt.

Der elektrische Energiebedarf wurde anhand von Messungen mit den verwendeten weitgehend aus marktüblichen Bauteilen zusammengestellten und nicht auf geringen elektrischen Energiebedarf optimierten - Magnetventilen ermittelt. Die Magnetspulen werden mit einer Spannung von $24 \mathrm{~V}$ versorgt. Für jede Magnetspule wird in der Öffnungsphase ein Boost-Strom von ca. 20A für eine Dauer von 1.5ms benötigt. Während der darauf folgenden Haltephase wird ein deutlich geringerer Strom von ca. 5.6A für die Magnetspulen der Einlassseite und 3.7A für die Magnetspulen der Auslassseite erforderlich. Dieser Haltestrom fließt entsprechend der Öffnungsdauer der Ventile. Für den Rest des Zyklus sind die Magnetspulen stromlos.

In der niedrigen Teillast kann der Reibmitteldruck des hier vorgestellten vollvariablen Ventiltriebs durch die Verwendung geringer Ventilhübe und kurze Öffnungsdauer reduziert werden, was mit einer konventionellen Nockenwelle nicht möglich ist. Über diesen Unterschied hinaus können durch eine entsprechende Wahl von Ventilhub und Öffnungsdauer Gaswechselverluste nahezu vermieden werden, was mit einer konventionellen Nockenwelle ebenfalls nicht möglich ist.

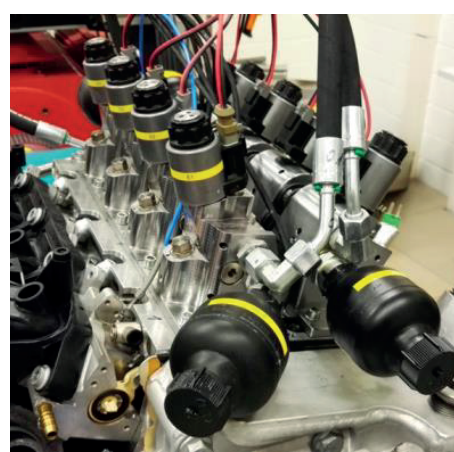

Abbildung 4: Foto des

Prüfstandsaufbaus.

\section{Vorläufige experimentelle Ergebnisse}

Der Versuchsmotor ist ein Volkswagen 1.4 TSi (EA111) Vierzylindermotor, welcher im Originalzustand sowohl durch ein Roots- wie auch durch einen Turbolader aufgeladen wird. Der Turbolader wurde nicht aufgebaut, da das Hauptinteresse der Untersuchungen dem Teillastbetrieb gilt. Der Kompressor ist allerdings weiterhin verbaut und kann z.B. für einen möglichen Zweitaktbetrieb ein positives Spülgefälle bereitstellen.

Der Motor und der Ventiltrieb werden mit einem auf einer dSpace MicroAutoBox basierenden Rapid-Prototyping-Umgebung gesteuert 
und geregelt. Dieser Ansatz ermöglicht ein Höchstmaß an Flexibilität um alle notwendigen Funktionalitäten sicherzustellen und alle Sensoren und Aktuatoren anzusteuern. Ein externes Hydraulikaggregat, welches unabhängig vom Motor betrieben werden kann, stellt die Hydraulikversorgung bereit. Eine serientaugliche Hydraulikversorgung, wie beispielsweise vom Motor angetriebene Pumpen, stand nicht im Fokus dieses Projekts. Abbildung 4 zeigt ein Foto des Prüfstandsaufbaus.

Zum Abgabezeitpunkt dieses Artikels war der Versuchsmotor noch nicht betriebsbereit. Die Einlass- und Auslassmodule waren allerdings montiert und die Gaswechselventile konnten unabhängig vom drehenden Motor betätigt werden.

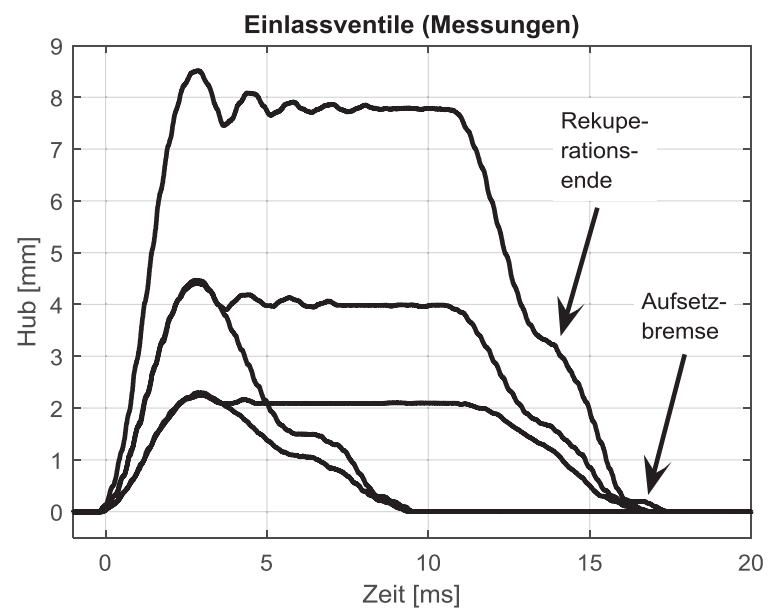

Abbildung 5: Gemessene Hubkurven der Einlassventile, verschiedene Ventilhübe und Öffnungsdauern (es ist nicht die kürzest-mögliche Bestromungsdauer gezeigt).
Im Folgenden zeigen einige Messresultate die Eigenschaften des Ventiltriebs auf, sowohl im stationären Betrieb mit verschiedenen Ventilhüben und Öffnungsdauern als auch die Änderung von Hydraulikdruck und Ventilöffnungsdauer für transiente Vorgänge.

\section{Stationärer Motorbetrieb}

Abbildung 5 zeigt die Ventilhubkurven der Einlassventile für verschiedene Hydraulikdrücke und Bestro-

mungsdauern. Unabhängig von Druck und Bestromungsdauer wird der maximale Ventilhub stets nach $3 \mathrm{~ms}$ erreicht. Nach einer kürzeren oder längeren Haltephase, während welcher der Ventilhub leicht oszilliert, schließen die Ventile während 4ms. Nach etwas mehr als der Hälfte des Schließvorgangs ist je ein kleiner „Höcker“ in den Hubkurven zu sehen. Schließlich setzen die Ventile in den letzten zehntel Millimetern des Schließvorgangs sanft auf, mit einer Geschwindigkeit von $0.3 \mathrm{~m} / \mathrm{s}$.

Die Öffnungszeit ist wie zuvor beschrieben unabhängig vom Ventilhub. Die Oszillationen in der Haltephase ergeben sich durch die Kompressibilität des Hydraulikfluides. 
Der Höcker im Schließvorgang resultiert aus dem Rekuperationsende und das sanfte Aufsetzen der Ventile ist durch eine hydraulische Aufsetzbremse gegeben. Entsprechend der Auslegung beträgt die Öffnungsdauer $7 \mathrm{~ms}$, was beispielsweise bei $5000 \mathrm{U} / \mathrm{min} 210^{\circ} \mathrm{KW}$ Ventilöffnung entspricht. Der Druckbereich für einen Hub von $0.5 \mathrm{~mm}-9 \mathrm{~mm}$ reicht von 44-120bar.

\section{Transienter Motorbetrieb}

Um den Verbrennungsmotor transient betreiben zu können, müssen der Ventilhub und die Öffnungsdauer der Gaswechselventile verändert werden. Im Folgenden wird dies für die Einlassseite gezeigt; für die Auslassseite funktioniert das Gezeigte analog.

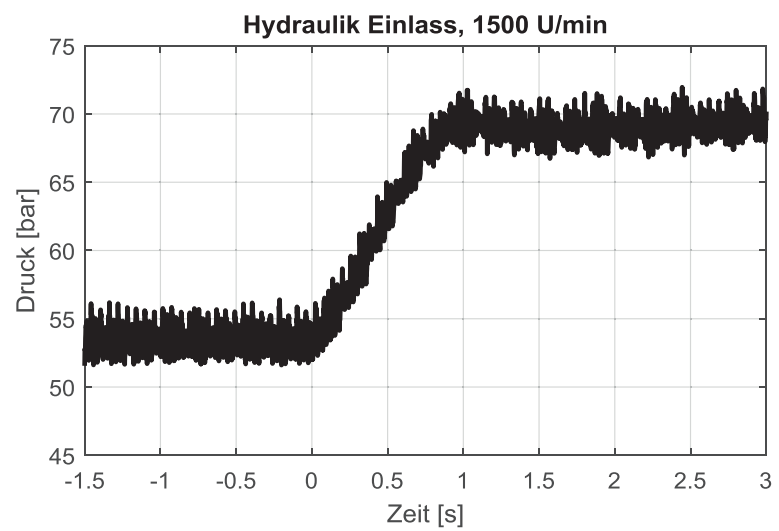

Abbildung 6: Erhöhung des Hydraulikdrucks einlassseitig.

Abbildung 6 zeigt eine Erhöhung des Hydraulikdrucks der Einlassseite von 54bar auf 70bar. Der Ventilhub ändert sich dabei von $1.7 \mathrm{~mm}$ auf $3.5 \mathrm{~mm}$.

Die Druckerhöhung benötigt knapp 1s, die Dynamik ist eher langsam. Grund dafür ist der zur Abdämpfung von Druckwellen verwendete Membranspeicher, welcher im Vergleich zur verwendeten Pumpe sehr groß ist. Die Größe des Speichers hängt direkt mit der Zeit für die Druckänderung zusammen, d.h. eine Reduktion der Größe führt zu einem schnelleren Druckaufbau. Ebenso würde eine stärkere kurzzeitige Überhöhung des Pumpenvolumenstroms den Druckaufbau beschleunigen.

Der linke Subplot in Abbildung 7 zeigt den gemessenen Öffnungs- und Schließzeitpunkt im Betrieb eines Einlassventils. Die Steuerzeit „Einlass schließt“ wird nach spät verstellt und danach wieder etwas nach früh. Der rechte Subplot zeigt alle dabei gemessenen Ventilhubkurven während der gesamten Messdauer. Die eingetragenen Zahlen zeigen die Zusammengehörigkeit der verschiedenen Bereiche im linken und 
rechten Subplot an. Die Messkurven zeigen deutlich, dass diese Verstellung von einem Zyklus auf den nächsten möglich ist.
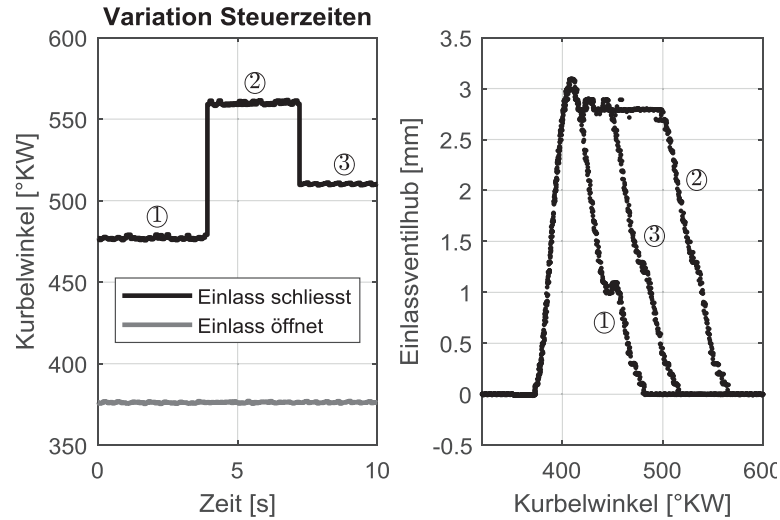

Abbildung 7: Messungen zur Änderung der Einlass-schließtSteuerzeit.

können, wird im nachfolgenden Kapitel beschrieben.
Zusammengefasst bedeutet das, dass es für die Lastregelung einen schnellen und einen langsamen Pfad gibt. Der langsame Pfad ist die Erhöhung des Einlassventilhubes (Erhöhung des Hydraulikdrucks), der schnelle Pfad die Änderung der Ventilöffnungsdauer (Bestromungsdauer der Magnetspulen). Wie diese beiden Möglichkeiten für die Lastregelung optimal kombiniert werden

\section{Lastregelung}

In diesem Kapitel wird die Lastregelung mit dem vollvariablen Ventiltrieb vorgestellt. Beispielhaft wird dies für eine Motordrehzahl von 1500U/min gezeigt, bei anderen Drehzahlen kann allerdings die gleiche Vorgehensweise angewendet werden. Hier werden - anders als zuvor - Simulationsergebnisse gezeigt. Die stationären Ergebnisse des Motorbetriebs sind mit einem 1D Modell berechnet worden, die transienten Ergebnisse mit einem 0D Modell, welches mit den Ergebnissen der 1D Simulationen erstellt wurde.

Schwerpunktmäßig wird in diesem Kapitel erklärt, wie die beiden Freiheitsgrade des Einlassventilhubs und der Öffnungsdauer der Einlassventile dafür ausgenutzt werden können die Motorlast anzupassen. Die anderen vier Freiheitsgrade „Einlass öffnet“", „Auslass öffnet“, „Auslass schließt“" und Auslassventilhub sind hierbei stark vereinfacht gewählt: 
Steuerzeiten und Ventilhübe

\begin{tabular}{|l|l|}
\hline Freiheitsgrad & Wert(e) \\
\hline Einlassventilhub & Variiert: $0.5-4.5 \mathrm{~mm}$ \\
\hline Öffnungsdauer Einlass & Ca. $60^{\circ}-240^{\circ} \mathrm{KW}$ \\
\hline Einlass öffnet & $0^{\circ}$ nach OT \\
\hline Auslass öffnet & $30^{\circ}$ vor UT \\
\hline Auslass schließt & $30^{\circ}$ nach OT \\
\hline Auslassventilhub & min. $2.5 \mathrm{~mm}$ oder gleich wie der Einlassventilhub \\
\hline
\end{tabular}

Den Autoren ist bewusst, dass für einen optimalen Motorbetrieb in allen Betriebspunkten die Steuerzeiten nicht überall gleich gewählt werden können, allerdings liegt

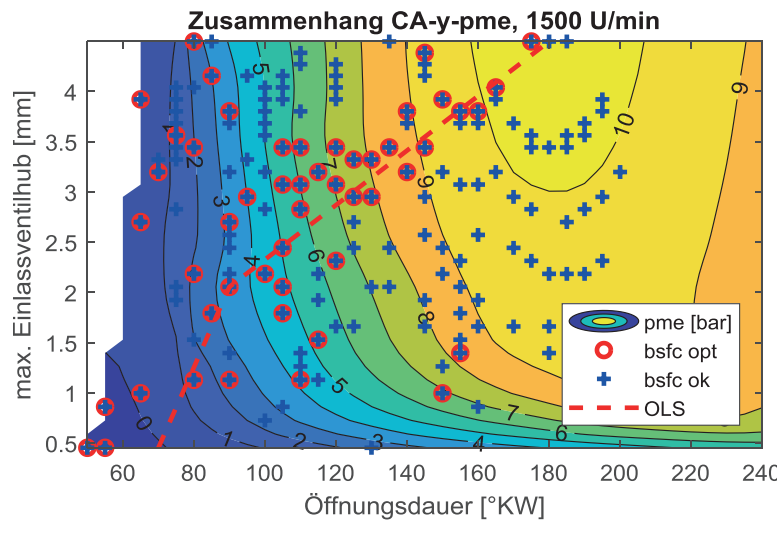

Abbildung 8: Effektiver Mitteldruck in Abhängigkeit von Einlassventilhub und Öffnungsdauer. Die Punkte zeigen wo der beste/gute Verbrauchswerte erzielt werden und in rot dargestellt der optimalen Lastschnitt (OLS). der Fokus in diesem Kapitel wie bereits erwähnt nicht auf der Optimierung der Verbrennung, sondern auf der Ausnutzung der beiden erwähnten Freiheitsgrade für die Lastregelung. Die Optimierung der anderen Freiheitsgrade kann unabhängig von der hier gezeigten Methodik durchgeführt werden und beeinflusst das Konzept zur Regelung der Motorlast nicht.

Abbildung 8 zeigt den effektiven Mitteldruck für eine Variation von Einlassventilhub und Öffnungsdauer im jeweils erwähnten Bereich. Die mechanische Reibung des Motors und der mechanische und elektrische Energiebedarf des Ventiltriebs ein- und auslassseitig sind in diesen Ergebnissen berücksichtigt. Der erreichte effektive Mitteldruck liegt hierbei zwischen -0.9bar (befeuerter Schub) und 10.3bar im nicht-aufgeladenen Betrieb. Tendenziell führen eine längere Öffnungsdau- 
FlexWork - Lastregelung bei einem Motor mit elektrohydraulischem, vollvariablem ...

er und ein größerer Ventilhub zu einer Erhöhung des effektiven Mitteldrucks, der gezeigte Bereich entspricht den Steuerzeiten des Miller Kreisprozesses. Für sehr lange Öffnungsdauern nimmt der effektive Mitteldruck wieder ab (Atkinson Kreisprozess, nicht dargestellt).

Die roten Kreise zeigen, in welchen Betriebspunkten der Verbrauch für verschiedene effektive Mitteldrücke minimal ist. Die blauen Marker zeigen, wo der Verbrauch maximal 2\% schlechter ist als der Minimalverbrauch. Für längere Öffnungsdauern muss die Bestromungsdauer erhöht werden, was zu einem höheren elektrischen Energiebedarf führt. Für größere Ventilhübe muss der Hydraulikdruck erhöht werden, was zu einem höheren Energiebedarf führt. An dieser Stelle wird einerseits klar, wie der Energiebedarf des Ventiltriebes und die Lastregelung zusammenhängen. Andererseits zeigt sich auch deutlich, dass bei kurzen Bestromungsdauern und wenig Einlasshub, sprich bei geringen effektiven Mitteldrücken in der niedrigen Teillast, der Energiebedarf des Ventiltriebs am geringsten ist.

Um den Lastschnitt für diese Drehzahl zu bestimmen muss eine Betriebslinie in dieses Kennfeld gelegt werden, die möglichst nahe an allen verbrauchsoptimalen Betriebspunkten liegt. Dieser optimale Lastschnitt ist in Abbildung 8 in rot eingetragen. Gezeigt ist ein bilinearer Ansatz, der über drei Punkte definiert ist. Allerdings muss angemerkt werden, dass auch diverse andere Linienverläufe für den optimalen Lastschnitt möglich wären. Die einzige Bedingung ist, dass sämtliche Mitteldrücke durchlaufen werden und jeder Wert nur einmal vorkommt.

Im stationären Motorbetrieb ergibt sich der Betriebspunkt des Ventiltriebs über den Schnittpunkt des optimalen Lastschnitts mit dem gewünschten effektiven Mitteldruck. Im transienten Motorbetrieb kann bei langsamen Laständerungen der Betriebspunkt auf dem optimalen Lastschnitt gehalten werden über Anpassungen des Hydraulikdrucks und der Öffnungsdauer. Für schnelle Änderungen der Motorlast kann natürlich - anders als bei herkömmlichen Ventiltrieben - mit der Verstellung der Öffnungsdauer eine sehr schnelle Anpassung erfolgen, was im Folgenden genauer erläutert wird.

In Abbildung 8 ist ersichtlich, dass entlang des optimalen Lastschnitts sowohl der Hydraulikdruck (also der Einlassventilhub) als auch die Öffnungsdauer angepasst werden müssen. Abbildung 6 und Abbildung 7 zeigen auf, dass die Verstellung des Hubes nur langsam erfolgen kann, während eine Verstellung der Öffnungsdauer innerhalb von $180^{\circ} \mathrm{KW}$ möglich ist. Wird der Motor in einem Betriebspunkt in der niedrigen Teillast betrieben, so kann bei gleichem Einlassventilhub durch eine Verlängerung der Öffnungsdauer der effektive Mitteldruck bereits im nächsten feuernden Zylinder sehr stark erhöht werden. Das bedeutet, dass durch ein kurzzeitiges Abweichen vom optimalen Lastschnitt eine deutliche Verbesserung der Dynamik möglich ist. 

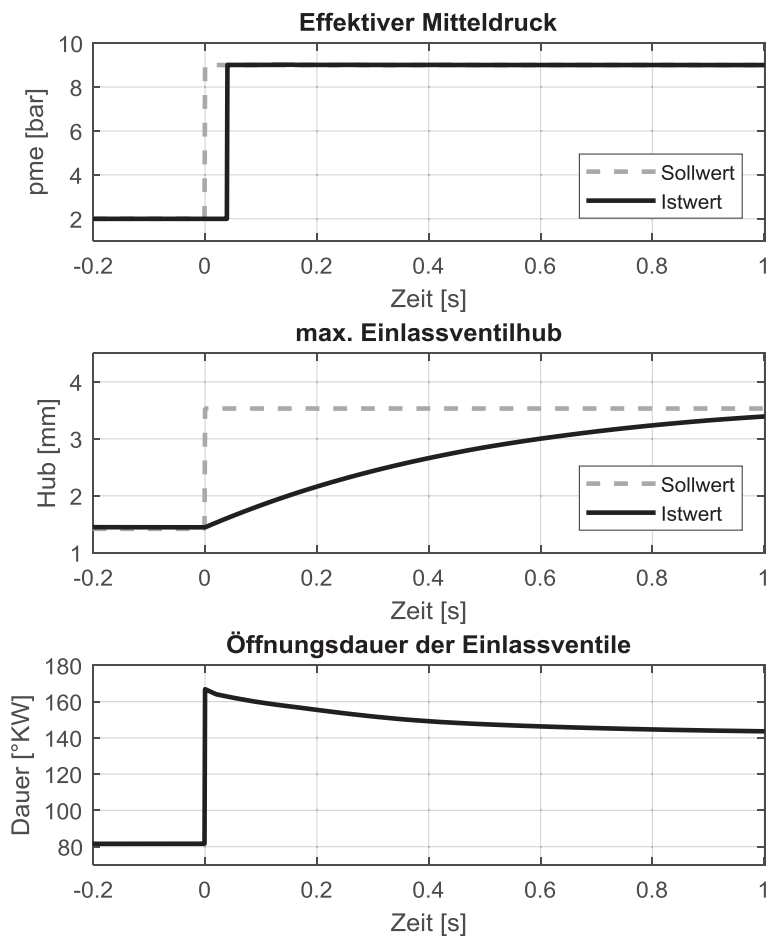

Abbildung 9: Lastsprung von 2bar auf 9bar effektiven Mitteldruck mit transienter Kompensation.

wird, öffnen die Einlassventile wieder weniger lang.
Ein Tip-In Vorgang für $1500 \mathrm{U} / \mathrm{min}$ ist in Abbildung 9 dargestellt. Der obere Subplot zeigt, dass der Sollwert der Last zum Zeitpunkt 0s von 2 bar auf 9bar effektiven Mitteldruck erhöht wird. Der Istwert der Last folgt - ebenfalls sprungförmig - mit einer Verzögerung von $0.04 \mathrm{~s}$. Der mittlere Subplot zeigt den Einlassventilhub, der langsam innerhalb von etwa $1 \mathrm{~s}$ seinen neuen Sollwert erreicht. Der untere Subplot zeigt die Öffnungsdauer der Einlassventile. Sobald der Lastsprung erfolgt, öffnen die Einlassventile deutlich länger. Während der Einlassventilhub langsam auf den neuen Sollwert erhöht

Es ist also möglich, die Motorlast mit einer Verzögerung von nur $0.04 \mathrm{~s}$, was einer Motorumdrehung entspricht, zu erreichen. Diese Verzögerung entspricht der Totzeit vom Ansaugen des Gemisches bis zum Expansionstakt und ist unumgänglich. Die Tatsache, dass der Einlassventilhub nur sehr langsam ansteigt, ist auf die verwendeten Membranspeicher zurückzuführen und könnte, wie bereits erwähnt, sehr einfach mit einem kleineren Speicher verbessert werden. Die schnelle Anpassung der Öffnungsdauer stellt sicher, dass die Motorlast sehr schnell angehoben werden kann, obwohl der Einlassventilhub in den ersten Millisekunden nach dem Sollwertsprung praktisch unverändert ist. Mit steigendem Einlassventilhub wird die Öffnungsdauer wieder zu- 
rückgenommen. Dafür wird im Kennfeld interpoliert, welche Öffnungsdauer beim momentanen Einlassventilhub zur gewünschten Motorlast führt. Diese Abweichung vom optimalen Lastschnitt wird als „transiente Kompensation“ bezeichnet.

Eine andere Darstellung von Einlassventilhub und Öffnungsdauer während der transienten Kompensation ist in Abbildung 10 zu sehen. Sie zeigt den Verlauf des Motorbetriebspunktes wäh-

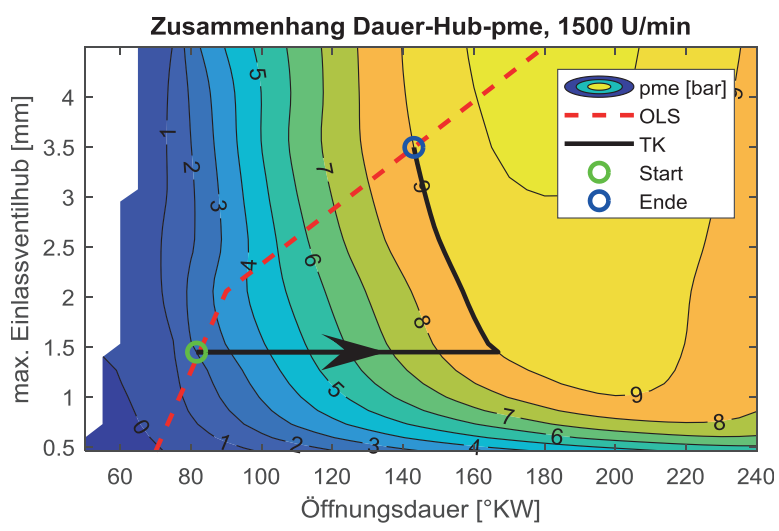

Abbildung 10: Transiente Kompensation (TK) für einen Lastsprung von 2 bar auf 9bar pme bei 1500U/min. Start- und Endpunkt sind auf dem optimalen Lastschnitt, während der Transienten weicht der Betriebspunkt vom optimalen Lastschnitt (OLS) ab. rend der Transienten im eingangs des Kapitels vorgestellten Betriebskennfeldes des Motors. Start und Ende der Transienten sind in der Abbildung markiert, die Betriebspunkte während der transienten Kompensation liegen auf der schwarzen Kurve.

In dieser Abbildung wird ersichtlich, dass sämtliche anderen Lastsprünge zu anderen effektiven Mitteldrücken ebenfalls auf diese Weise durchgeführt werden können.

Es muss lediglich die entsprechende Öffnungsdauer aus dem Kennfeld ausgelesen werden.

Da eine derart schnelle Erhöhung der Motorlast in der Regel unerwünscht ist, kann dies mit verschiedenen Methoden künstlich verlangsamt werden. Eine Möglichkeit wäre beispielsweise, die maximal zulässige Änderungsrate des effektiven Mitteldruckes zu limitieren. Abbildung 11 zeigt die transiente Kompensation für drei verschiedene Änderungsraten im Vergleich. Der linke Subplot zeigt die Verläufe des effektiven Mitteldrucks, der rechte Subplot die dazugehörigen Verläufe der transienten Kompensation. Der abgestufte Verlauf kommt daher, dass für diese Simulationen ein

diskretes Motormodell verwendet wurde. Alle $0.02 \mathrm{~s}\left(180^{\circ} \mathrm{KW}\right)$ resultiert ein neuer Wert für den effektiven Mitteldruck. Die Abbildung zeigt sehr deutlich, dass künstlich verlangsamte Lastsprünge mit der vorgestellten Methodik kein Problem darstellen. 
Der rechte Subplot zeigt, dass sich der Verlauf der transienten Kompensation bei langsameren Änderungsraten zunehmend dem optimalen Lastschnitt tatsächlich annähert.
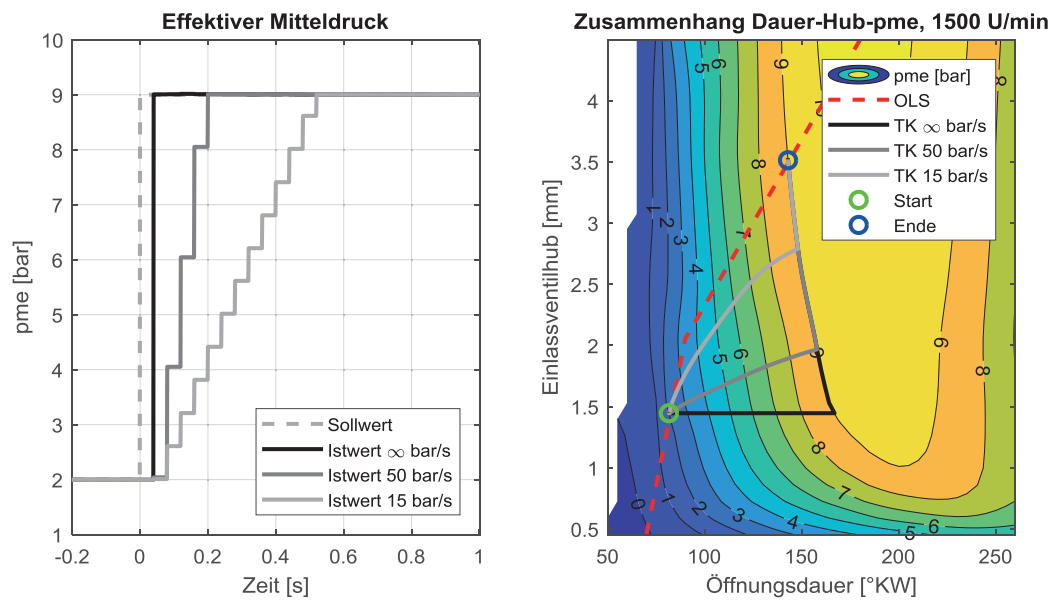

Abbildung 11: Transiente Kompensation (TK) für einen Lastsprung von 2 bar auf $9 \mathrm{bar}$ bei 1500U/min mit verschiedenen Änderungsraten für den effektiven Mitteldruck.

Abschließend noch einige Anmerkungen zu der hier vorgestellten Methodik. Ein Lastabwurf kann auf genau die gleiche Weise realisiert werden. Dort wird zunächst die Öffnungsdauer reduziert, während der Einlassventilhub abnimmt. Die Betriebspunkte der transienten Kompensation sind in diesem Fall oberhalb des optimalen Lastschnittes. Die Erhöhung und die Verringerung der Last sind nicht nur für Miller Steuerzeiten auf die gezeigte Weise möglich, sondern auch für lange Öffnungsdauern (Atkinson Kreisprozess). Zu guter Letzt noch eine Anmerkung zur Optimierung der Steuerzeiten: Selbst, wenn die Optimierung der Steuerzeiten im gesamten Kennfeld zu großen Unterschieden zu den hier verwendeten Steuerzeiten führt, ändert das nichts an der Funktionsweise der hier vorgestellten Methodik. Die Konstant-pme-Linien bekommen durch die Optimierung der Steuerzeiten einen anderen Verlauf, was lediglich die Lage des optimalen Lastschnittes beeinflusst. Die Methodik wurde auch für diese Fälle in der Simulation getestet und funktioniert weiterhin, sowohl für Miller als auch für Atkinson Steuerzeiten und sowohl für Lasterhöhungen als auch für Lastabwürfe. 
FlexWork - Lastregelung bei einem Motor mit elektrohydraulischem, vollvariablem ...

\section{Energiebedarf}

Der Energiebedarf eines vollvariablen Ventiltriebs ist ein wichtiges Kriterium für eine Bewertung des Systems, wird aber nur selten thematisiert. In diesem Abschnitt werden der elektrische und der mechanische Energiebedarf des vorgestellten FlexWork Ventiltriebs mit dem eines nockengetriebenen Ventiltriebs verglichen.

Die Berechnung des mechanischen Energiebedarfs des Ventiltriebs wurde in Kapitel 2 beschrieben. Für die hydraulische Versorgung wurde eine regelbare Hydraulikpumpe mit einem konstanten Wirkungsgrad von 85\% angenommen, die Rekuperation entsprechend der am jeweiligen Betriebspunkt verwendeten Ventilhubkurven berücksichtigt. Der elektrische Energiebedarf wurde wie in Kapitel 2 beschrieben quantifiziert.

Die Berechnung des Energiebedarfs des FlexWork Ventiltriebs erfolgt unter Annahme eines worst-case Szenarios. Einerseits könnte der elektrische Energiebedarf der Magnetventile, die aus marktüblichen Einzelteilen zusammengestellt wurden, noch optimiert werden, andererseits wird die Auslassseite stets so betrieben, dass die Auslassventile bis zu einem Zylinderdruck bei „Auslass öffnet" von 10bar öffnen könnten. Vor allem in der niedrigen Teillast ist der Zylinderdruck bei „Auslass öffnet“ deutlich niedriger als 10bar. Eine Anpassung der Ansteuerung des Auslass würde also den hydraulischen Energiebedarf weiter senken.

Für den konventionellen Ventiltrieb ist die Annahme, dass unabhängig von Drehzahl und Motorlast stets $1 \mathrm{~J}$ pro Ventilöffnung und Ventil erforderlich sind. Bei 16 Ventilen und einem Hubraum von 1.39Litern ergibt sich ein Reibmitteldruck von 0.1151bar (bzw. 1.27Nm Reibmoment). Darüber hinaus wird ein konstantes Reibmoment von $0.5 \mathrm{Nm}$ für die Steuerkette angenommen. Um diese Annahmen zu stützen sind hier einige veröffentlichte Ergebnisse zu dem Thema zusammengetragen. Der Reibmitteldruck von Nockenwelle und Nocken ist für das Auslassventil eines 0.6Liter 1Zylinder Dieselmotor in [6] mit 0.058-0.067bar angegeben (3.5-4J/Öffnungsvorgang). In [7] wird das Reibmoment der Auslassnockenwelle für einen 0.5Liter 1-Zylinder Motor mit 2 Auslassventilen mit 1.2Nm angegeben. Dies entspricht einem Reibmitteldruck von 0.3bar. Die Studie zeigt auch, dass das Reibmoment der Auslassnockenwelle im befeuerten Betrieb um 10-14\% zunimmt gegenüber dem geschleppten Betrieb. Für den hier gezeigten Vergleich ist dieser Effekt nicht berücksichtigt. Messungen in [8] zeigen, dass die Gleitreibung der Steuerkette selbst bei einer niedrigen Drehzahl von 2000U/min einen Anteil von 16\% an der gesamten Motorreibung ausmacht. Die hier getroffenen Annahmen für den Energiebedarf eines Nockentriebs sind also sehr optimistisch. Auch der Energiebedarf eines Mechanismus zur Phasenverstellung der Nockenwellen ist nicht berücksichtigt, aber in Realität durchaus relevant. In [9] wird gezeigt, dass ein Wechsel von einer hydraulischen zu einer elektrischen Vorrichtung zur Phasenverstellung eine Einsparung von 125-300W über den 
NEFZ Fahrzyklus aufgrund einer kleineren Ölpumpe mit sich bringt. Mit den verwendeten Annahmen besteht also für den nockengetriebenen Ventiltrieb ein best-case Szenario.
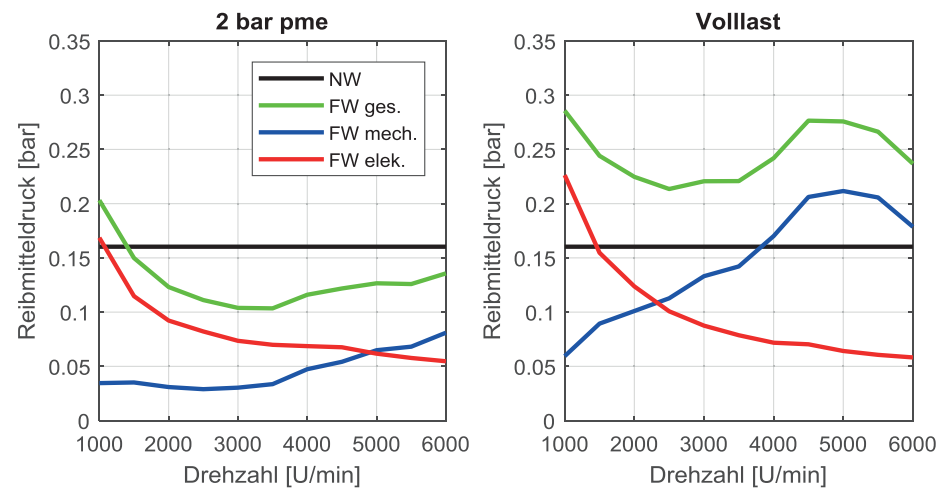

Abbildung 12: Reibmitteldruck des Ventiltriebs. NW zeigt die Werte für einen nockengetriebenen Ventiltrieb, FW für hier den vorgestellten FlexWork Ventiltrieb, aufgeteilt in den mechanischen Anteil und den elektrischen Anteil (jeweils Einlass- und Auslassseite).

Abbildung 12 zeigt die Werte für den gesamten Reibmitteldruck des Ventiltriebs (einlass- und auslassseitig) für verschiedene Motordrehzahlen. Zu sehen ist der Vergleich für eine mechanische Nockenwelle (NW) und den FlexWork Ventiltrieb (FW). Bei letzterem ist auch die Aufteilung in mechanischen und elektrischen Energiebedarf abgebildet. Der linke Subplot zeigt, dass in der Teillast der mechanische Energiebedarf des FlexWork Ventiltriebs über alle Drehzahlen hinweg deutlich geringer ist als der eines nockengetriebenen Ventiltriebs. Der elektrische Energiebedarf steigt mit abnehmender Drehzahl an. Der Gesamtenergiebedarf des FlexWork Ventiltriebs ist außer bei sehr niedrigen Drehzahlen geringer als der eines nockengetriebenen Ventiltriebs. Der rechte Subplot zeigt, dass der FlexWork Ventiltrieb an der Volllast mechanisch mehr verbraucht als in der Teillast und bei hohen Drehzahlen auch mehr als ein nockengetriebener Ventiltrieb. Der elektrische Energiebedarf nimmt auch an der Volllast für niedrigere Drehzahlen zu. Der Maximalwert für die gesamte Reibleistung beim FlexWork Ventiltrieb beträgt $1.7 \mathrm{~kW}$ bei $5000 \mathrm{U} / \mathrm{min}$.

Der Grund für den niedrigen mechanischen Energiebedarf des FlexWork Ventiltriebs in der Teillast ist, dass nur ein kleiner Ventilhub notwendig ist um den Gaswechsel zu gewährleisten. Der niedrige elektrische Energiebedarf bei hohen Drehzahlen ist darauf zurückzuführen, dass die Bestromungsdauer sehr nahe an der Mindestbestromungs- 
dauer liegt und es nur eine sehr kurze Haltephase für den Strom gibt. Bei niedrigen Drehzahlen hingegen folgt auf die Mindestbestromungsdauer noch eine lange Haltephase um die Ventile offen zu halten. An der Volllast ist mit zunehmender Motordrehzahl ein größerer Ventilhub erforderlich um eine gute Füllung zu ermöglichen, was höhere Hydraulikdrücke und somit ein Mehr an mechanischem Energiebedarf bedeutet. Der elektrische Energiebedarf ist an der Volllast bei hohen Drehzahlen ähnlich wie in der Teillast, da die Bestromungsdauer auch hier nahe an der Mindestbestromungsdauer liegt. Bei niedrigen Drehzahlen steigt der elektrische Energiebedarf noch leicht an, da für die Volllast eine längere Haltephase nötig ist als für die Teillast.

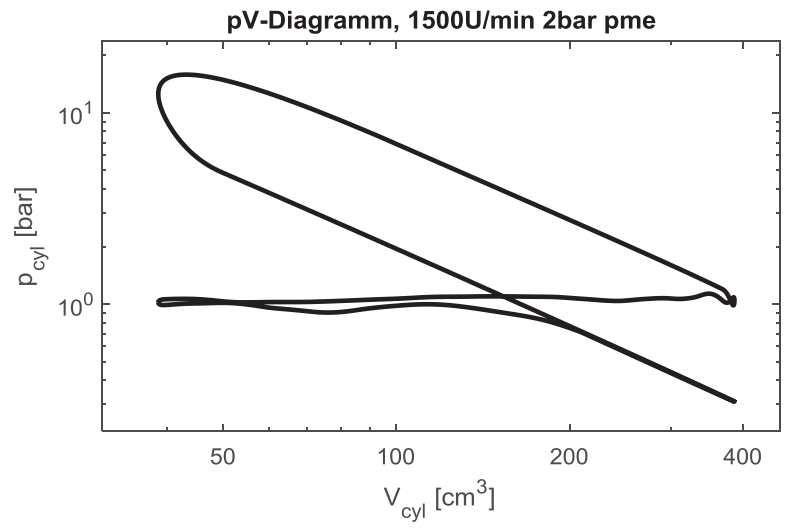

Abbildung 13: pV-Diagramm in der niedrigen Teillast bei einer Motordrehzahl von 1500U/min.
Diese Ergebnisse zeigen, dass der FlexWork Ventiltrieb in der Teillast geringfügig weniger Energie benötigt als ein nockengetriebener Ventiltrieb, und das obwohl ein best-case-szenario für die Nockenwelle mit einem worst-caseSzenario für den FlexWork Ventiltrieb verglichen wurde. An der Volllast ist der Energiebedarf des FlexWork Ventiltriebs höher als für nockengetriebene Systeme, aber diese Betriebspunkte sind zum einen nicht oder nur wenig zyklusrelevant und zum anderen könnte hier der höhere Energiebedarf mit einem optimierten Gaswechsel durch die Freiheitsgrade des Ventiltriebs überkompensiert werden.

Über diese Betrachtungen hinweg darf die Verbesserung des Ladungswechsels natürlich nicht in Vergessenheit geraten. Abbildung 13 zeigt das doppellogarithmische pVDiagramm einer Simulation für 2bar effektiven Mitteldruck bei 1500U/min. Die Ladungswechselverluste für diesen Punkt in der niedrigen Teillast betragen gerade einmal 0.048bar.

Der FlexWork Ventiltrieb benötigt also dort wenig Energie, wo es beim typischen Einsatz im PKW am wichtigsten ist: in der Teillast. Und zusätzlich dazu können eben- 
falls in der Teillast die Ladungswechselverluste mit dem FlexWork Ventiltrieb drastisch verringert werden.

\section{Zusammenfassung und Ausblick}

Ein vollvariabler Ventiltrieb ist vorteilhaft für die Optimierung sämtlicher Betriebsbereiche eines Verbrennungsmotors. Der hier vorgestellte elektrohydraulische FlexWork Ventiltrieb vereint die Vorteile einer geringen Systemkomplexität mit großer Variabilität und einem geringen Energiebedarf. Der Betrieb mit Motorkühlmittel führt zu einem ölfreien Zylinderkopf, was weniger additiviertes Motoröl und eine erhöhte Katalysatorlebensdauer mit sich bringt.

Der Energiebedarf des Systems hängt vom Ventilhub und von der Öffnungsdauer der Ventile ab, wobei kleine Ventilhübe und kurze Öffnungsdauern zu einem geringen Energiebedarf führen.

Eine Methodik für die Lastregelung unter der Verwendung der beiden Freiheitsgrade Einlassventilhub und Öffnungsdauer wurde im Detail erklärt. Mit dem vorgestellten FlexWork Ventiltrieb ist ein exzellenter transienter Motorbetrieb möglich, in dem von einer Ventilöffnung auf die nächste die Öffnungsdauer beliebig verstellt wird, während der Hydraulikdruck (Ventilhub) entsprechend des neuen Betriebspunktes angepasst wird. So sind zum einen sehr schnelle Lastsprünge möglich, zum anderen sind Geschwindigkeit und Verlauf einer Drehmomentänderung sehr frei wählbar.

Anhand eines Lastschnitts bei 2bar effektivem Mitteldruck und an der Volllast wurde der Energiebedarf mit einer konventionellen Nockenwelle verglichen. In der Teillast ergibt sich sogar für eine worst-case Betrachtung des vorgestellten FlexWork Ventiltriebs ein kleiner Vorteil im Vergleich zum Energiebedarf einer konventionellen Nockenwelle. Anhand eines pV-Diagramms bei 1500U/min und einem effektiven Mitteldruck von 2 bar wird aufgezeigt, dass sich selbst in der niedrigen Teillast sehr geringe Ladungswechselverluste ergeben: 0.048bar.

Für den weiteren Projektverlauf sind die Inbetriebnahme des Versuchsmotors im befeuerten Betrieb, die Implementierung der Lastregelung am Prüfstand, der Moduswechsel zum 2-Zylinderbetrieb sowie Untersuchungen des 2-, 6-, 8-Takt Betriebs geplant.

\section{Danksagung}

Die Autoren bedanken sich bei folgenden Partnern, welche wesentlich zum Gelingen des Projektes beigetragen haben: Beim schweizerischen Bundesamt für Energie (BFE) Sektion Energieforschung für die finanzielle Unterstützung sowie bei den Firmen Muhr und Bender KG (D-Attendorn), Elgo Electronic GmbH (D-Rielasingen- 
FlexWork - Lastregelung bei einem Motor mit elektrohydraulischem, vollvariablem ...

Worblingen), Hydac International GmbH (D-Sulzbach/Saar), Bieri Hydraulik AG (CH-Liebefeld) und bei der BASF SE (D-Ludwigshafen).

\section{Literaturverzeichnis}

[1] R. Van Basshuysen, Handbuch Verbrennungsmotor. Springer Vieweg Wiesbaden, Germany, 2017.

[2] P. Leduc, B. Dubar, A. Ranini, and G. Monnier, "Downsizing of Gasoline Engine: an Efficient Way to Reduce CO 2 Emissions," Oil Gas Sci. Technol. - Rev. IFP, vol. 58, no. 1, pp. 115-127, 2003.

[3] L. Guzzella, Modeling and control of internal combustion engine systems. Springer: Berlin Heidelberg, Germany, 2010.

[4] H. Wei, T. Zhu, G. Shu, L. Tan, and Y. Wang, "Gasoline engine exhaust gas recirculation - A review," Appl. Energy, vol. 99, no. X, pp. 534-544, 2012.

[5] O. A. Kutlar, H. Arslan, and A. T. Calik, "Methods to improve efficiency of four stroke, spark ignition engines at part load," Energy Convers. Manag., vol. 46, no. 20, pp. 3202-3220, 2005.

[6] M. Teodorescu, D. Taraza, N. Henein, and W. Bryzik, "Experimental Analysis of Dynamics and Friction in Valve Train Systems," SAE Tech. Pap. Ser. 2002-010484, no. 724, 2002.

[7] R. A. Mufti and M. Priest, "Experimental Evaluation of Engine Valve Train Friction under Motored and Fired Conditions," Tribol. Res. Des. Eng. Syst., vol. 41, pp. 767-778, 2003.

[8]T. Hyakutake, M. Inagaki, M. Matsuda, N. Hakamada, and Y. Teramachi, "Measurement of friction in timing chain," JSAE Rev., vol. 22, no. 3, pp. 343347, 2001.

[9] J. Schäfer, J. Balko, "High performance electric camshaft phasing system," SAE Tech. Pap., vol. 2007, no. 724, pp. 01-1294, 2007. 
Norbert Zsiga, Andyn Omanovic, Patrik Soltic

Empa, Swiss Federal Laboratories

for Materials Science and Technology

Überlandstrasse 129

8600 Dübendorf

Switzerland

Wolfgang Schneider

Wolfgang Schneider Ingenieurbüro

3600 Thun,

Switzerland 